\title{
Editorial
}

\section{The impact of recent control of asbestos regulations upon the building surveying industry}

\author{
Journal of Building Appraisal (2008) 4, 49-53. doi:10.1057/jba.2008.21
}

Asbestos is a naturally occurring mineral, with deposits occurring all over the world. There are even geological outcrops of asbestos to be found in South East England, however commercial extraction of asbestos is now limited to Canada and Russia. The use of asbestos as a building material has been banned in the UK since 1999, but asbestos was used extensively from the 1950s, to manufacture a wide range of building materials. There is therefore an unknown but potentially massive amount of asbestos fibres present within many buildings built pre-millennium. Asbestos has in fact been used by man since pre-history. Its name derives from the Greek word asbest, which means unquenchable and relates to its quality of being fireproof. Asbestos is a fibrous mineral, which can be found naturally in one of two forms, serpentine or amphilbole. The former relates commercially to chrysotile (white asbestos), while the latter relates commercially to crocidolite (blue asbestos) and amosite (brown asbestos). The colour-coded names relate to the appearance of the material in its natural state, but this colour tends to change during processing, making identification of the type of asbestos impossible outside of scientific testing. While chrysolite is recognised as being of lower risk to health than crocidolite or amosite, the risk with all is significant, and asbestos-containing materials (ACMs) when sampled are often found to contain more than a single type of asbestos fibre.

Asbestos is a very versatile material. It has strong tensile strength, good insulating properties, high levels of durability and resists heat up to temperatures of around 1,400 degrees C. It can be made into many different types of materials, including rigid boarding, flexible textiles, sprayable foam, lightweight cements, durable tiling, mouldable pastes and moulded components. It is rare for an ACM to be exclusively formed from asbestos fibres and the 3,000 plus products found to contain asbestos tend to have differing percentages of asbestos within them.

While links between direct contact with asbestos fibres and premature deaths have been noted since early times it was not until the early 20th century that the first case of lung fibrous, now known as asbestosis, was officially recorded in the UK, and not until the 1930s that the first regulatory control on asbestos use was introduced. Exposure to asbestos fibres can result in a number of untreatable and generally fatal diseases. The most widely known is asbestosis. This occurs when a fibre of asbestos becomes embedded in the tissue wall of the lungs. In trying to remove the immovable fibres the respiratory defence mechanism releases chemicals to break down the asbestos fibres. While having no effect on the asbestos fibre these chemicals damage the lungs, reducing its capacity over a period of time. Other noted asbestos-related diseases include contribution to lung cancer, particularly among smokers where asbestos exposure is estimated to increase the likelihood of death by lung cancer by up to 50-fold. Mesothelioma is a cancer attributed specifically to asbestos exposure, and forms a cancerous tumour at the point that an 
asbestos fibre passes through the wall of the lung, or abdomen. A further nonfatal but debilitating disease pleural plaques reduces lung capacity but does not in itself progress to a fatal condition. It must be noted that dangerous contact with asbestos mostly occurs when an ACM becomes damaged or disturbed, thus releasing the fibres into the air to be inhaled or ingested.

Exposure to airborne asbestos fibres is deemed responsible for contraction of the previously cited diseases. This can occur during extraction of the raw asbestos, the manufacture of asbestos products, installation of asbestos products or the degradation of those products in service, but latterly the most likely method of exposure is the disturbing of an ACM during works to a building. This might include cutting into, grinding, drilling or repair/replacement of damaged elements. Consequentially the top of the list of occupations deemed most at risk of exposure to asbestos is almost exclusively filled by those engaged in the property-based trades of construction, demolition, building maintenance, mechanical and electrical services and surveying.

This places those engaged in the appraisal of buildings directly in harm's way, both professionally and personally. The Control of Asbestos at Work Regulations 2002 (CAWR 2002), and Control of Asbestos Regulations 2006 (CAR 2006), place specific conditions in respect of the management of asbestos, which include the identification and establishing of ACMs within a building. The nature of the surveying process often requires access to those areas where previously undiscovered ACMs are most commonly found. This requires the surveyor to be aware of the risks attributable to disturbing ACMs, and to be mindful of the obligations imposed by the asbestos control legislation, namely CAWR 2002 and CAR 2006.

CAWR 2002 established the position of a single or dual duty holder with the statutory responsibility to manage the risks of exposure to asbestos fibres within a specified building (Figure 1). As this duty holder must have a contractual responsibility regarding the repair and maintenance of the building this would rarely be a building surveyor, however the professional responsibilities between the surveyor and the client would mean that the terms of this legislation will also impact upon the building surveyor. These terms include the taking of reasonable steps to identify the location and condition of ACMs, which would be a task mostly undertaken by the appropriately trained building appraiser/ surveyor. Any materials suspected of potentially containing asbestos must be presumed as doing so. This puts a requirement on surveyors to be able to identify the estimated 3,000 plus asbestos-containing products in all of the potential uses that each may be employed (Figure 2). Consequential to the discovery of a potential ACM, steps must be taken to record its location, assess its potential risk, devise an appropriate plan for negating any possibility of exposure to asbestos fibres, pass relevant information on to anyone who it may put at risk and keep a constant watching brief over the ACM until it is either removed from the premises or declared by scientific testing to not contain any asbestos fibres. Many of these tasks require the skills and knowledge of a professional surveyor, however overall responsibility for preventing exposure cannot be devolved from the duty holder to the surveyor.

CAR 2006 developed the terms of the previous legislation into a more prescriptive set of instructions. It introduced the need for a specific asbestos register to be kept permanently available for inspection whenever required. This register is not only to be the repository for information on the location of all identified and suspected ACMs within the building, but also requires detailed plans of the building. There is now an obligation for the duty holder to arrange for inspections to monitor the condition of known ACMs and establish any further suspected material. This is part of a more proactive management 


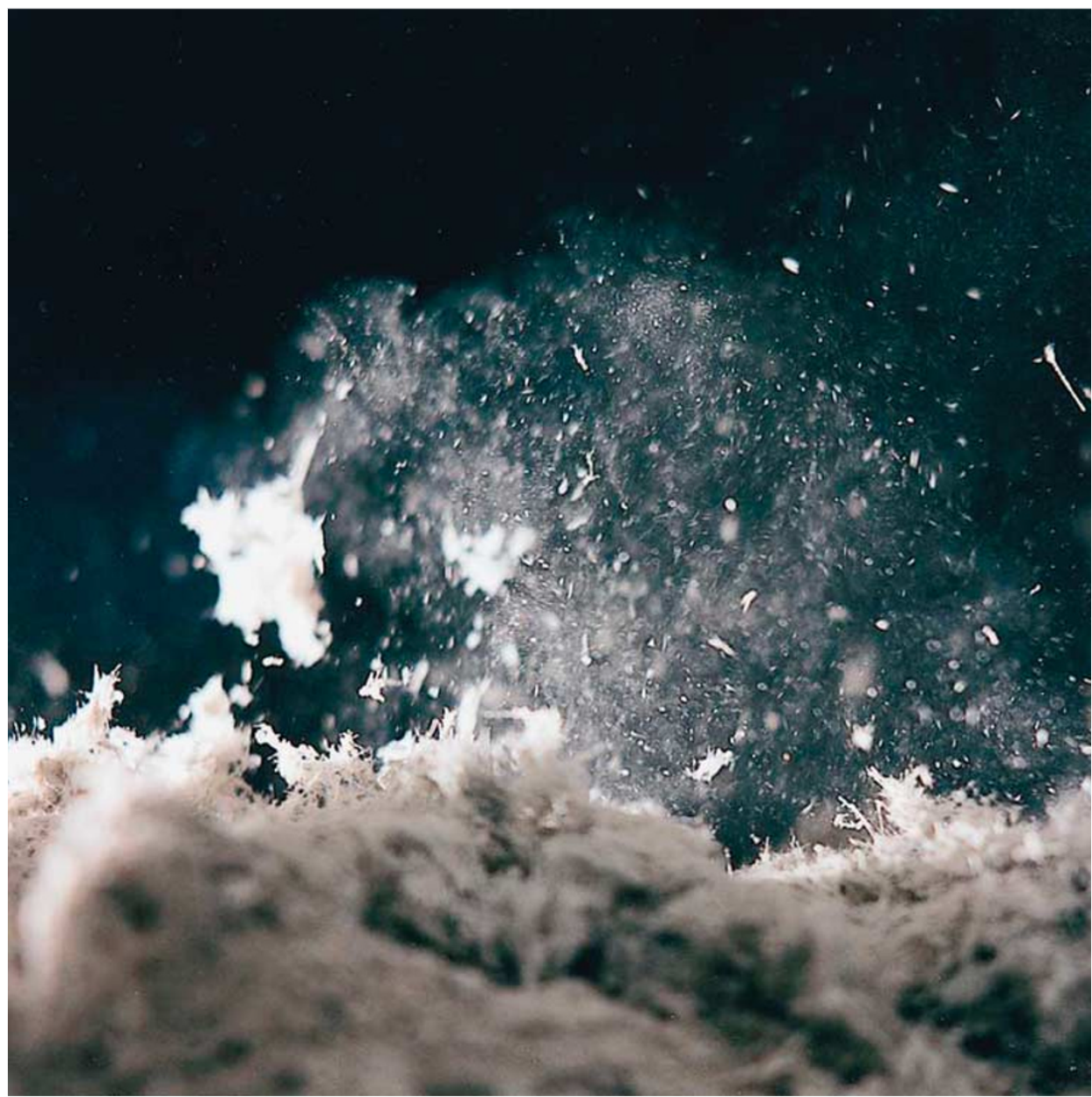

Figure I: Asbestos fibres from insulation material are easily disturbed

plan, which would require greater technical input as decisions on the best way to deal with asbestos-related risks are made. The provisions of this management plan are to be communicated to anyone who could potentially disturb a known or suspected ACM. Three types of physical asbestos survey are noted within the statute, which are Type 1 (visual inspection only), Type 2 (visual inspection and sampling) and Type 3 (invasive inspection), dismantling where required and sampling. The first would generally fall within the remit of a trained surveyor, however, the latter two due to the direct contact with samples of potential ACMs require specially trained and equipped surveyors. Finally, CAR 2006 requires the monitoring and maintenance of health records for any employee, surveyors included, who are inadvertently exposed to the risk of contact with asbestos fibres within the building.

The prescriptive nature of these asbestos control regulations require an increased awareness of ACMs and the risks they impose and also dictate a change of focus within the traditional building survey. They additionally create a more specialist form of survey specifically targeted at identification, risk assessment and management of asbestos. This means that the need to identify and control asbestos-related risk has a much greater professional impact on the work of a surveyor, but also awareness of risks created by the actual and potential presence of asbestos impacts upon working practices. Thus, asbestos 


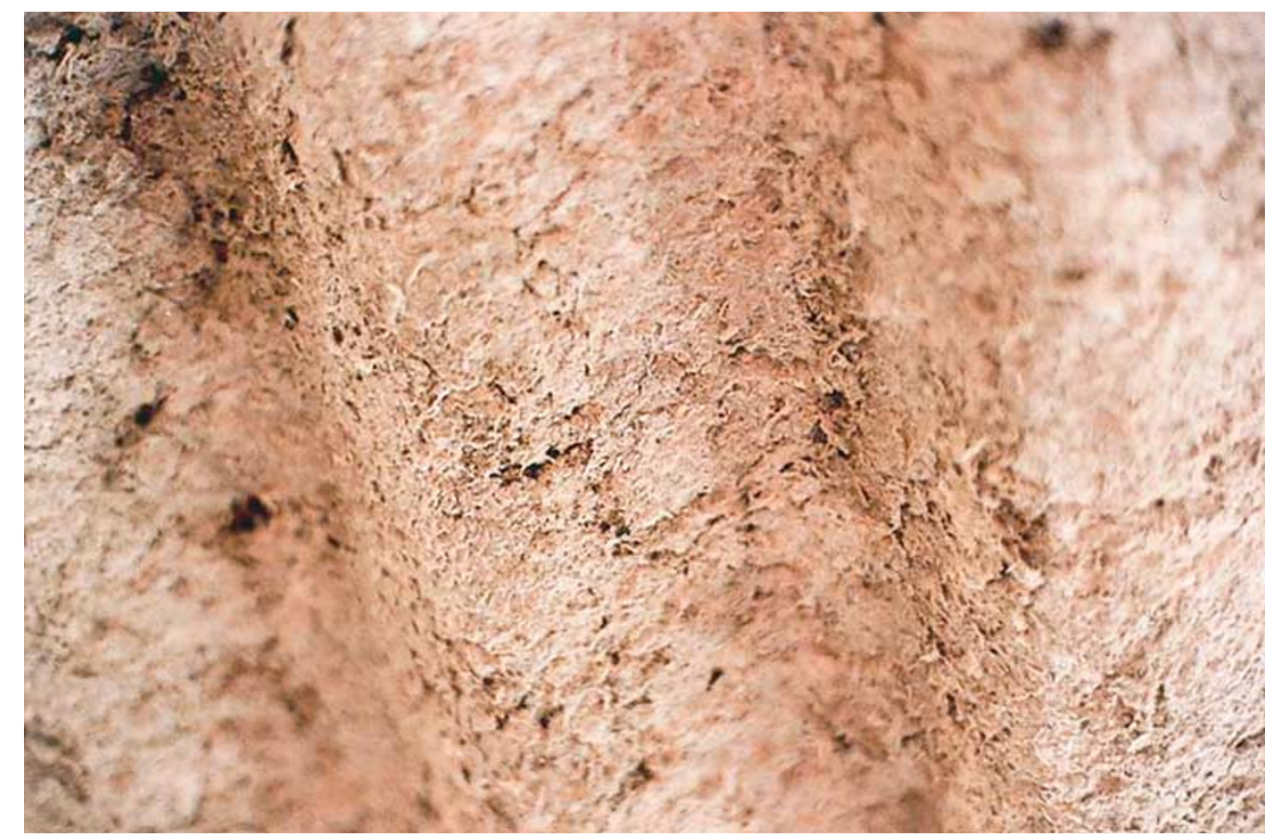

Figure 2: Asbestos-containing material used to provide fire proofing to exposed steel work

awareness training, currently vital for those engaged in at risk occupations, covers both the identification of ACMs and also how to avoid disturbing asbestos fibres. Simple examination mediums such as the Royal Society for the Promotion of Health's Certificate in asbestos awareness test both knowledge of identification of potential ACMs and an ability to work in a way that minimises risk of direct contact with any airborne asbestos fibres.

The working practices of a building surveyor must have changed. HSE guidelines identify working on an unfamiliar site as a major factor in adding to the risk of exposure to asbestos, and this is often a key feature of the daily work of the typical building surveyor. Therefore before engaging in a survey of a commercial premises the surveyor should refer to the asbestos register, which must be kept available as part of compliance with CAR 2006. Apart from the good practice aspect of reference to detailed plans of the building to be surveyed this is required in order for the surveyor to become acquainted with the location and condition of any previously discovered ACMs. It is also required that the surveyor while working is able to comply fully with the terms of any previously established and written management plan for not disturbing asbestos fibres within those premises. Where required a surveyor must use appropriate personal protection equipment, especially respiratory protection, and localised hygiene practices, to ensure that any inadvertent contact with asbestos fibres is contained. Commonly used methods of testing for such common building defects as fungal decay can involve drilling to provide access for an endoscope or minor dismantling. These techniques should now be used sparingly, and with greater care, and the use of power tools be replaced where possible with manually driven tools. It must be remembered that the activity of the surveyor must not only put them at risk but also anyone who might follow into the working area. Any material that might contain asbestos fibres must be treated as doing so until scientific testing proves otherwise. When specifying work which may require breaking into areas where asbestos might be found, that is, voids between floors and partitioning, the 
commissioning of a Type 3 asbestos survey may be required to be included as part of the specification before the actual building work commences.

It could be argued that there has never been a greater single impact on the work of those engaged in appraising the condition of buildings than the recently heightened awareness of the risks associated with asbestos and the consequential asbestos control legislation. The presence of ACMs is possible and often likely in any building built before 1999. There are fixed duties imposed under criminal law upon those who manage commercial buildings, and direct reference within those statutes to management tasks that require the skills of professional surveyors. Consequentially there is a requirement for those surveyors during every survey as a vital part of their work to identify potential ACMs and provide appropriate advice in respect of their treatment and management. As one of the occupations considered to be at greatest risk of exposure to asbestos, the current surveyor is required to participate fully in delivering any management plans for working amidst a potential risk of releasing asbestos fibres, while being mindful of personal protection in the event of the discovery of suspected ACMs. Surveyors need to be particularly aware of the risk to anyone who follows into areas where rigorous testing methods for building defects analysis have been employed, and the need to exclude others from areas where suspected ACMs are found. Given the numbers of buildings which might potentially contain asbestos, and the nature of the tests prescribed by statute, many building surveyors find that asbestos work takes up an ever increasing part of their work, and some of them now work exclusively in identifying and managing ACMs.

Simon Mclean

Managing Editor 